\title{
EFFECT OF DRY RELAXATION ON LENGTHWISE MEASUREMENT DEVIATION OF POLYESTER/SPANDEX PLATED KNITTED TUBES AFTER DYEING
}

\section{RAMEESH LAKSHAN BULATHSINGHALA}

\author{
Department of Textile and Clothing Technology, University of Moratuwa, Sri Lanka
}

\begin{abstract}
Weft knitted fabrics produced in a circular knitting machine undergo dry relaxation as soon as fabric is removed from the constraints of the machine. A significant deviation of lengthwise (wale direction) measurements was observed among the tubular articles knitted first and last within the same batch. Different elongations at heat-setting determined the final lengthwise measurement after dyeing. The deviation in lengthwise measurement due to dry relaxation at heat-setting resulted in a significant measurement variation after dyeing. Due to this reason, during the bulk production, a considerable portion of garments are not within the tolerance and ultimately increase a higher percentage of rework and rejections. This paper investigates on how dry relaxation is correlated with the lengthwise measurements of polyester/spandex blend plated knit tubes during heat-setting and dyeing. The relationship between thermoelastic behaviour and dry relaxation of the specimens was also studied. The increase in temperature from $22^{\circ} \mathrm{C}$ to $32^{\circ} \mathrm{C}$ accelerated the dry relaxation, and garments reached to an equilibrium condition in 10 hours with a significant improvement in relaxation time than that of 115 hours at $22^{\circ} \mathrm{C}$. The increase in temperature brought a higher proportion of garment within the fluctuation of $1.5 \mathrm{~cm}$ after dyeing. This research also addresses the importance of considering dry relaxation curve while giving measurement adjustments in the pilot run.

KEYWORDS: Dry Relaxation, Heat-Setting, Knitting, Lengthwise Measurement, Plating, Polyester/Spandex \& Thermal Shrinkage
\end{abstract}

Received: May 30, 2020; Accepted: Jun 19, 2020; Published: Jul 11, 2020; Paper Id.: IJMPERDJUN2020286

\section{INTRODUCTION}

Yarn plating in a circular knitting machine is a promising technique to produce elastic knit fabrics for sportswear and outerwear to improve the recovery performance. In the plating technique, elastic spandex yarn is fed through a plating feeder together with a high-modulus yarn either cotton, polyester or nylon in the knitting process to form a plated loop and hence produce a plated fabric. With plating, the hard yarn and the spandex yarn are knitted parallel, side-by-side relation, with the spandex yarn always kept on one side of the hard yarn, and hence on one side of the knitted fabric. Due to the strain energy developed in the structure during the knitting process, knit loops become more resilient and hence lead to relaxation by recovering the stress during the subsequent processes. At the relaxed stage, compressed stitches in the knitted article are at an equilibrium condition between spandex recovery forces and resistance to stitch compression by the companion hard yarn. In this research, polyester/spandex half plated tubular knitted articles were used to assess the effect of dry relaxation on lengthwise measurement after heat-setting and dyeing process.

After knitted fabric is released from the machine, the stress developed in the fabric structure is released, and fabric will retract to compress the loops by reducing the dimensions. This stress relaxation process is called as dry relaxation. Senthilkumar et al. (2012) investigated the stretch and recovery behaviour of stretch fabrics. The 
researcher mentioned that dry relaxation process reduces the potential distortion and deformation of the knit fabric from the residual stress developed during knitting. The input tension of the spandex yarn, linear density of the spandex yarn, loop length of the hard yarn are the major variables which govern the elastic recovery of the plated fabrics. The loop length is considered as the primary knitted structure parameter which determines the physical properties such as fabric porosity, tightness factor, stitch compression, stretch recovery and dimensional stability (Eltahan et al., 2016; Hurd \& Doyle, 1953; Pavko-Cuden et al., 2013; Prakash \& Thangamani, 2010; Senthilkumar et al., 2011, 2012). Moreover, the loop length in the weft knitted articles is influenced by yarn input tension, take down tension, yarn physical properties, machine gauge, knitting velocity, knit structure, knitting friction, etc. (Lau et al., 1994; Pavko-Cuden et al., 2013). Hurd \& Doyle (1953) mentioned that stitch density at dry relaxed state, "jam point," is closely related to stitch length and unaffected by other knitting and yarn variables.

In dry relaxation, knitting construction and type of yarn determine the strain-free conditions of the fabric (Munden, 1959). The ability of stress recovery (tensile resilience) depends on the yarn type (Munden, 1959). Bandari et al. (2020) analysed the effect of fabric structure and strain percentage of rib weft knitted articles on the lengthwise stress relaxation. The author stated that friction between the yarns in the structure and the space for free movement of loops are the two determinant factors of stress relaxation and experimentally showed that increase in tuck stitches leads to subsequent increase in stress relaxation.

Dry relaxation is a time-dependent process. Fatkić et al. (2011) stated that yarn input tension should be optimized for an effective relaxation since a low strain on the yarn will make fabric reach equilibrium soon with a minimum potential energy. Bandari et al. (2020) incorporated tuck loops in the pattern to accelerate the relaxation. Nevertheless, yarn input tension and knitting pattern determine the physical properties of the structure and hence performance of the fabric; therefore, there are constraints in changing the knitting parameters to control the dry relaxation. For this reason, in the knitting industry, manufactures use relaxation machines to relax knitted fabrics. Dynamic dry relaxation is a promising technique used for relaxation of knitted fabric by using machines such as C-Tex and ST-FRM, and previous studies have also focused in developing fast fabric relaxing machines (Jayawardana et al., 2016). However, instead of machines, knitted articles in tubular form are relaxed on a flat smooth surface in the standard atmospheric condition $\left(22^{\circ} \mathrm{C}, 65 \%\right.$ R.H $)$. This process is called as static dry relaxation.

During the static dry relaxation process, production time is a crucial parameter which determines the relaxation shrinkage of the garments within the batch. In this context, there is a significant measurement deviation in the wale direction (length direction) between the articles which were knitted first and last. The experimental results showed a measurement difference of $8 \mathrm{~cm}$ in the wale direction. Due to this reason, knitted tubes have different elongations at the heat-setting process. The experimental results showed that elongation at heat-setting determines the lengthwise measurement after dyeing.

Heat-setting of knitted fabrics which composed of thermoelastic materials serves to release the stress developed during the knitting process. The heat-setting operation therefore improves the stability of the fabric and reduces the amount that fabric will shrink after washing (Gupta, 2001). The heat-setting process which assists in improving dimensional stability and fibre morphology of thermoelastic materials depends on the heat-setting temperature, elongation of the fabric, duration of heat-setting and the heat-setting medium. In a polyester/spandex blend knit fabric, spandex undergoes strain induced crystallisation at heat-setting process, and elastic recovery properties change with the elongation at heat-setting. Crystallisation of spandex increases with the extension of yarn and therefore percentile of elasticity recovery changes during 
the heat-setting process. At optimum conditions, spandex materials under strain become stable at a new elongation by increasing the elastic modulus. However, thermal shrinkage behaviour of polyester material during heat-setting differs from spandex. Mody et al. (2001) and Perera \& Lanarolle, (2020) investigated the thermal shrinkage behaviour of polyester material after heat-setting and developed thermal shrinkage models and suggested effective parameters for heat- setting. These studies also revealed that thermal shrinkage increases with heat-setting temperature and extension ratio under specific conditions, which are discussed under the results and discussion section.

Sanjari et al. (2011) analysed seven relaxation methods for plain knitted fabrics and categorized them into three relaxation methods: mechanical relaxation, chemical relaxation and complex relaxation method. In this context, dry relaxation, wet relaxation, washing relaxation and ultrasonic waves relaxation were discussed under mechanical relaxation; detergent-washing and ultrasonic-chemical relaxation were discussed under complex relaxation method. The researcher concluded that chemical relaxation process has more effect on the dimension stability than all other mechanical relaxation processes. The researcher also introduced a novel relaxation method, ultrasonic-chemical relaxation, which was suggested as an effective method in terms of dimension stability and fabric regularity. Munden (1959) stated that products knitted with hydrophilic yarns can be bought to an equilibrium state in dimensions by relaxation in wet condition; the results were experimentally showed by immersing specimens in a water bath with a wetting agent for 12 hours. A similar experiment was conducted by Gowers \& Hurt (1978), and the researcher showed that agitation during tumble drying imparts a greater contribution for wet relaxation since tumbling contributes enough energy into fabric to overcome inter-frictional forces.

Although several authors have studied on mechanical and chemical relaxation methods, in a process sequence which consists of knitting, heat-setting and dyeing, there is a dearth of review in the literature analysing the effect of dry relaxation on lengthwise measurement during heat-setting and dyeing process. The experimental results of this research showed that there is a significant variation in the length measurement within the same batch due to dry relaxation. It was observed nearly $8 \mathrm{~cm}$ difference in the lengthwise measurement between the specimens which were knit first and last. This deviation resulted nearly $4 \mathrm{~cm}$ difference in lengthwise measurement after dyeing. Therefore, measurement deviations due to dry relaxation should be reduced to rectify the lengthwise measurement problems after dyeing. The measurement adjustment method from the pilot run to bulk production should also be changed since pilot knit tubes are produced within a short period than that of bulk. The bulk knit tubes in a batch undergo more relaxation than pilot knit tubes and therefore measurement adjustment should consider the relaxation curve of the bulk.

The scope of this research is to analyse the existing dry relaxation method and assess the effect of dry relaxation on lengthwise measurement after heat-setting and dyeing process. Therefore, an attempt has been made in this research to design a new methodology to regulate the measurement deviation of the knitted articles due to dry relaxation and hence overcome the measurement issues and variations within the batch after dyeing.

\section{MATERIALS AND METHODS}

\subsection{Material Specifications}

- Fibre composition: Polyester/Spandex

- Spandex tension: $4 \mathrm{cN}$

- Polyester tension: $6 \mathrm{cN}$ 
- Construction: Plain, half plating (plating elastane yarn in every second course of polyester yarn)

- Polyester count: 25Tex

- $\quad$ Spandex count: 40dTex

- $\quad$ Set length measurement(length): $85.5 \mathrm{~cm}$

\subsection{Material Preparation}

The tubular knitted articles were prepared by using the circular knitting machine with the specification shown in Table 1. A whole batch of $71 \mathrm{~kg}$ samples were prepared from polyester spun 30Tex and 40dTex sample. It was taken five days to produce the whole batch, and finished articles were laid flat in the bath preparation area for dry relaxation. The temperature and humidity of the conditioned room is $22^{\circ} \mathrm{C}$ and $65 \%$ respectively. Two machines were selected (machine 21 and machine 22 ) to produce the whole bulk.

Table 1: Knitting machine specifications

\begin{tabular}{|c|c|c|c|c|c|}
\hline Machine type & Model & Gauge & $\begin{array}{c}\text { Diameter } \\
\text { (inches) }\end{array}$ & No. of feeders & $\begin{array}{c}\text { Plating } \\
\text { technique }\end{array}$ \\
\hline Seamless circular knitting & Santoni SM8-TOP2 & npi 28 & 18 & 8 & Half plating \\
\hline
\end{tabular}

\subsection{Research Method}

Samples were placed on a smooth flat surface individually in a standard atmosphere $\left(22^{\circ} \mathrm{C}, 65 \%\right.$ R.H $)$ conditions until samples reach an equilibrium state in the length measurement (wale direction). The measurements of thirty garments were taken into account to determine the dry relaxation curve and the equilibrium state length measurement. Dry relaxation parameters were calculated by following equations.

Elongation $(e)$ at heat-setting= Before wash Length at heat-setting $\left(L_{l}\right)$ - Set length $\left(L_{0}=85.5 \mathrm{~cm}\right)$

Extension ratio $(\lambda)=\frac{L_{1}}{L_{0}}$

To analyse the effect of elongation on the measurement after heat setting, samples at different extensions were heat set. The heat-setting temperature and the duration were determined by analysing the existing literature. Previous studies showed that polyester fabrics are dimensionally stable if heat-setting temperature is higher by $30^{\circ} \mathrm{C}-40^{\circ} \mathrm{C}$ than the temperature to which fabric is subjected during subsequent processes (Ertekin \& Marmarali, 2016; Gupta, 2001; O'Dottavianoa at el., 2005). The glass transition temperature $\left(\mathrm{T}_{\mathrm{g}}\right)$ of polyester is approximately $80^{\circ} \mathrm{C}$, heat-setting temperature in the presence of dry air is between $\left(190^{\circ} \mathrm{C}-220^{\circ} \mathrm{C}\right)$ with the tension (Mody et al., 2001). Perera \& Lanarolle (2020) investigated the heat-setting temperature of polyester material in both hank and fabric form based on shrinkage and experimentally showed $160^{\circ} \mathrm{C}$ as an effective temperature for heat-setting of polyester in dry air. According to Gacén et al. (2002), heat-setting of polyester products is performed at temperatures from $160^{\circ} \mathrm{C}-220^{\circ} \mathrm{C}$ for $30-120$ seconds. The heatsetting conditions for stretch fabrics which incorporate spandex material varies from $180^{\circ} \mathrm{C}$ to $220^{\circ} \mathrm{C}$ in temperature for about 15 to 90 seconds (O’Dottavianoa at el., 2005). The-heat setting temperature was set at $180^{\circ} \mathrm{C}$ for about 45 seconds following the specifications mentioned in the Table 2. At this temperature, both polyester and spandex fabrics will be heat set. After heat-setting, the samples were allowed to cool down in the standard atmosphere for 24 hours to determine the thermal shrinkage due to het-setting process. Thermal relaxation due to shrinkage after heat-setting was calculated by following 
equation.

Thermal shrinkage after heat-setting $\left(S_{H T}\right)=$ Before wash Length at heat-setting $\left(L_{l}\right)$-measurement at equilibrium state after heat-setting $\left(L_{2}\right)$

\subsection{Heat-Setting Procedure}

Knitted tubes were heat set by using Technopia IR heat-setting machine, which is specially designed for heat-setting of tubular seamless products made of polyester material. Table. 2 represents the specifications of the heat-setting machine.

Table 2: Heat-setting specifications

\begin{tabular}{|l|l|l|c|c|}
\hline \multicolumn{1}{|c|}{$\begin{array}{c}\text { Machine } \\
\text { type }\end{array}$} & \multicolumn{1}{|c|}{ Technology } & \multicolumn{1}{|c|}{ Fibre type } & Board Size & $\begin{array}{c}\text { Maximum } \\
\text { température }\end{array}$ \\
\hline $\begin{array}{l}\text { IR heat- } \\
\text { setting } \\
\text { machine. }\end{array}$ & $\begin{array}{l}\text { Hot air heat-setting machine for } \\
\text { tubular products }\end{array}$ & $\begin{array}{l}\text { Polyester, Nylon, } \\
\text { Spandex }\end{array}$ & $35^{\prime \prime}-60^{\prime \prime}$ & $220^{\circ} \mathrm{C}$ \\
\hline
\end{tabular}

\subsection{Dyeing Procedure}

Samples were dyed in a sample dyeing machine at $130^{\circ} \mathrm{C}$ by using a disperse dyeing profile. Samples were allowed to cool in standard conditions for 24 hours before taking measurements. Dyeing shrinkage was calculated by the below expression.

Shrinkage after dyeing $\left(S_{A D}\right)=$ Before wash Length at heat-setting $\left(L_{1}\right)$ - After dyed measurement $\left(L_{3}\right)$

\section{RESULTS AND DISCUSSIONS}

\subsection{Dry Relaxation of Plated Knitted Articles}

After knitting, tubes were relaxed under standard atmospheric conditions until the whole batch was completed knitting prior to the next process, heat-setting. A significant deviation in lengthwise measurement was observed between the first and last portion of knitted tubes in the same batch. The Figure 1 shows a variation of $8 \mathrm{~cm}$ in length measurement between the first and last portion of the batch. After plating spandex yarn with the companion hard yarn, spandex takes more time to relax to its near original position due to the resistance of the hard yarn and the resistance of the structure. A loop in the knitted article formed by spandex does not relax to its near original position quickly as a normal spandex yarn due to the friction of other nearby stitches and resistance of the companion hard yarn. Therefore, during the dry relaxation, compressed loops in the knitted article reach to an equilibrium state where fabric becomes compact in length and width. This point is called as the "Jam point", and fabric will no longer relax beyond this point. When a plated fabric reached to a jam point, compressed stitches in the knitted article are at an equilibrium condition between spandex recovery forces and resistance to stitch compression by the companion hard yarn. According to Figure 1, the jam point measurement in length direction was approximately $86.5 \mathrm{~cm}$. However, the first $3 \mathrm{~cm}$ was recovered within first two hours because of the high strain energy and low resistance of the polyester yarn and structure. Even though polyester is the hard yarn, tensile resilience of polyester knit fabrics is excellent than other hard yarns. Therefore, shrinkage relaxation of polyester fiber is high at high extensions. The resilience of polyester/ spandex blend knitted fabrics is better than other compositions and make it suitable for sportwear and outerwear. 


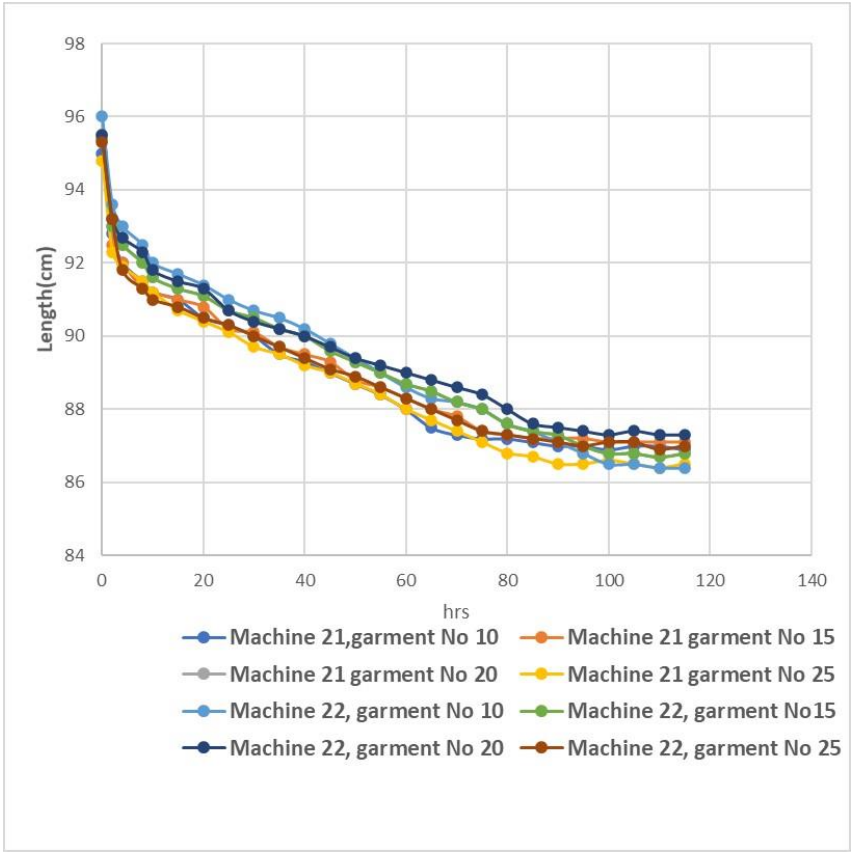

Figure 1: The change in lengthwise measurement due to relaxation

\subsection{Effect of Heat-setting on Thermal Shrinkage}

The thermal shrinkage $\left(S_{H T}\right)$ of the garments after heat-setting was increased with the increase in elongation. Polyester imparts a higher tensile resilience $(>70 \%)$ whether in treated or untreated form (Pavko-Cuden A at el., 2013). The researcher also concluded that increase in heat-setting duration increases the tensile resilience $\left(77.25 \%\right.$ of tensile resilience at $180^{\circ} \mathrm{C}$ for 240 seconds). However, $\mathrm{d} S_{H T} / \mathrm{d} e$ reduces with the increase in elongation which is attributed to the process involved in redeniering of spandex and thermal contraction behaviour of polyester.

Mody et al., (2001) stated five regions for thermal shrinkage of polyester films in the length direction. The experiment carried out at $100^{\circ} \mathrm{C}$ (beyond $\mathrm{T}_{\mathrm{g}}$ ) with the uniaxial draw ratios up to 3.75 . In region $\mathrm{I}$, shrinkage arises with the extension ratio due to contraction of tie molecules following a linear model. After contraction, chain molecules attain the orientation without developing any noticeable crystallinity level. In region II, shrinkage mechanism is same as in region I, but contracted tie molecules recrystallize by chain folding and hence increase crystallinity when extension ratios increase in region II. In addition, a decrease in shrinkage slope was observed with the increase in extention ratios in this region. In region III and IV, tie chains upon contraction become stain free and are then incorporated to crystallites; therefore, a drop in shrinkage was observed in this region with the increase in extension ratio. In region $\mathrm{V}$, the shrinkage is due to contraction of tie molecules and extended amorphous regions; parts of these extended amorphous regions are reformed into crystallites thus increasing the crystallinity and hence reduce the shrinkage. Perera \& Lanarolle (2020) analysed the thermal behaviour of polyester material in fabric due to heat-setting. Authors analysed the thermal shrinkage of polyester in hank and fabric form at different heat setting temperatures from $130^{\circ} \mathrm{C}$ to $200^{\circ} \mathrm{C}$ (Perera \& Lanarolle, 2020). The study concluded that increase in temperature increases the shrinkage during the heat-setting at temperatures above $160^{\circ} \mathrm{C}$; however, it was also noticed a thermal expansion in polyester fabric below $160^{\circ} \mathrm{C}$. This thermal expansion of polyester fabric was attributed to external forces exerted by the heat-setting machine which extended the fabric at heat-setting. Hence, the author concluded that effective temperature for heat-setting of polyester fabric is $160^{\circ} \mathrm{C}$. According to Figure 2, it was observed that thermal shrinkage behaviour of the specimens follows a similar pattern at extension ratios below 1.5 (elongation between $0.5 \mathrm{~cm}$ to $10.6 \mathrm{~cm}$ ) corresponding to 
region I and region II shrinkage curves. Moreover, specimens experienced an increase in thermal shrinkage with the increase in elongation Therefore, following the thermal shrinkage models proposed by Mody et al. (2001) and Perera \& Lanarolle (2020), it can be concluded that polyester material in the patted knitted tubes of this experiment follows a similar chacteristic shrinkage curve as proposed in region I and region II.

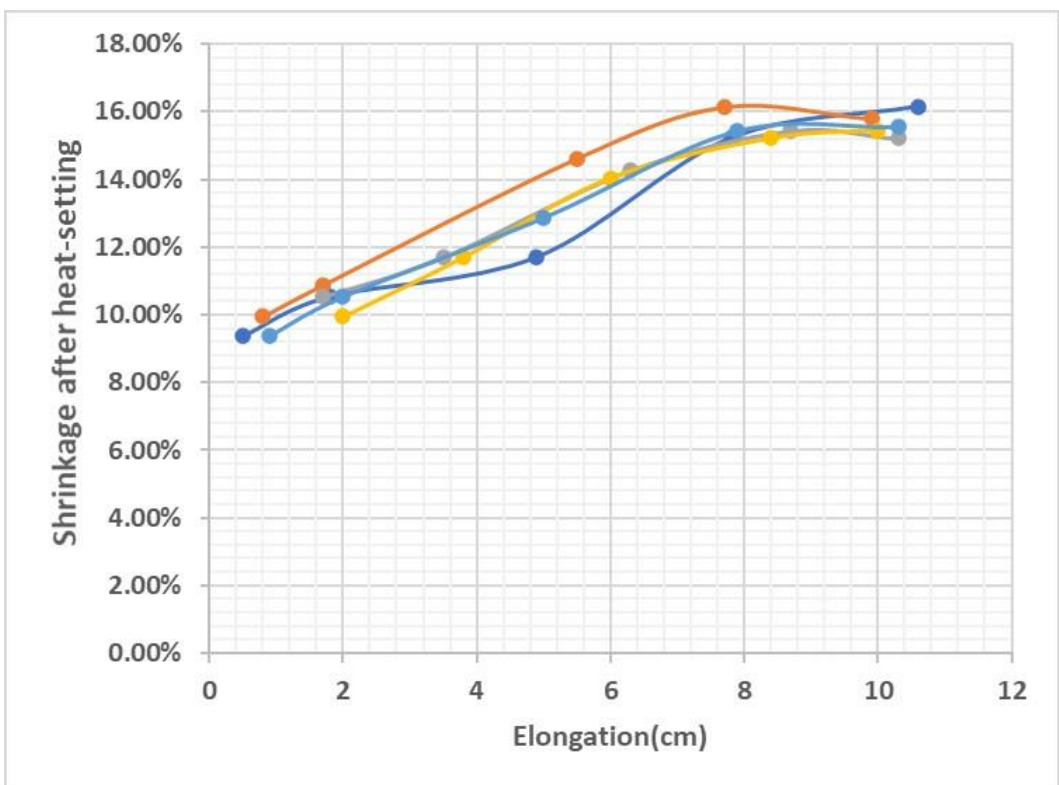

Figure 2: The effect of elongation on thermal shrinkage at heat-setting

During the heat-setting process, spandex yarn undergoes strain-induced crystallisation where extended soft segments create new crystallites. The elastic modulus changes due to the thermal melting of the crystallites and strain-induced crystalization. Elastic modulus at higher elongations increases during heat-setting since there is a permanent change at molecular level where inter molecules are broken and rearranged during the heat-setting process. Therefore, the recovery tension in the stretch fabric is revealed at a great proportion, and the compressed loops in the fabric tends to become stable at a new elongation.; this process is known as rendeniering. Because of the combination of redeniering process and thermal shrinkage behaviour of polyester, $S_{H T}$ increases with elongation and $\mathrm{d} S_{H T} /$ de decreases with elongation. With respect to all these explanations, it was observed that increase in elongation at heat-setting increases the thermal shrinkage after heat-setting. In Table 3, The maximum thermal shrinkage is $13.8 \mathrm{~cm}$ and minimum shrinkage is $8 \mathrm{~cm}$ after heat-setting.

Table 3: Experiment results

\begin{tabular}{|c|c|c|c|c|c|c|c|}
\hline $\begin{array}{c}\text { Measur } \\
\text { ement } \\
\text { at heat- } \\
\text { Setting( } \\
\mathbf{c m}) \boldsymbol{L}_{\mathbf{1}}\end{array}$ & $\begin{array}{c}\text { Measurement } \\
\text { after Heat- } \\
\text { setting at } \\
\text { equilibrium(cm) } \\
\boldsymbol{L}_{\mathbf{2}}\end{array}$ & $\begin{array}{c}\text { Thermal shrinkage } \\
\text { after heat- } \\
\text { setting(cm) } \boldsymbol{S}_{\boldsymbol{H T}}\end{array}$ & $\begin{array}{c}\text { Thermal } \\
\text { Shrinkage } \\
(\boldsymbol{\%}) \text { after } \\
\text { heat-setting }\end{array}$ & $\begin{array}{c}\text { Elongation } \\
(\mathbf{c m}) \boldsymbol{e}\end{array}$ & $\begin{array}{c}\text { Extension } \\
\text { ratio } \\
\boldsymbol{\lambda}\end{array}$ & $\begin{array}{c}\text { After Dyed } \\
\text { Measurement } \\
(\mathbf{c m}) \\
\boldsymbol{L}_{\mathbf{3}}\end{array}$ & $\begin{array}{c}\text { Shrinkage( } \\
\mathbf{c m})\end{array}$ \\
\hline 86 & 78 & 8 & $9.36 \%$ & 0.5 & 1.00585 & 58 & 28 \\
\hline 87.3 & 78.3 & 9 & $10.53 \%$ & 1.8 & 1.02105 & 58.3 & 29 \\
\hline 90.4 & 80.4 & 10 & $11.70 \%$ & 4.9 & 1.05731 & 60.4 & 30 \\
\hline 93.3 & 80.3 & 13 & $15.20 \%$ & 7.8 & 1.09123 & 60.3 & 33 \\
\hline 96.1 & 82.3 & 13.8 & $16.14 \%$ & 10.6 & 1.12398 & 62.3 & 33.8 \\
\hline 87.2 & 78.2 & 9 & $10.53 \%$ & 1.7 & 1.01988 & 58.2 & 29 \\
\hline 89 & 79 & 10 & $11.70 \%$ & 3.5 & 1.04094 & 59 & 30 \\
\hline 91.8 & 79.6 & 12.2 & $14.27 \%$ & 6.3 & 1.07368 & 59.6 & 32.2 \\
\hline 94.2 & 81 & 13.2 & $15.44 \%$ & 8.7 & 1.10175 & 61 & 33.2 \\
\hline 95.8 & 82.8 & 13 & $15.20 \%$ & 10.3 & 1.12047 & 62.8 & 33 \\
\hline
\end{tabular}




\begin{tabular}{|c|c|c|c|c|c|c|c|}
86.4 & 78.4 & 8 & $9.36 \%$ & 0.9 & 1.01053 & 58.4 & 28 \\
\hline 87.5 & 78.5 & 9 & $10.53 \%$ & 2 & 1.02339 & 58.5 & 29 \\
\hline 90.5 & 79.5 & 11 & $12.87 \%$ & 5 & 1.05848 & 59.5 & 31 \\
\hline 93.4 & 80.2 & 13.2 & $15.44 \%$ & 7.9 & 1.09240 & 60.2 & 33.2 \\
\hline 95.8 & 82.5 & 13.3 & $15.56 \%$ & 10.3 & 1.12047 & 62.5 & 33.3 \\
\hline 86.3 & 77.8 & 8.5 & $9.94 \%$ & 0.8 & 1.00936 & 57.8 & 28.5 \\
\hline 87.2 & 77.9 & 9.3 & $10.88 \%$ & 1.7 & 1.01988 & 57.9 & 29.3 \\
\hline 91 & 78.5 & 12.5 & $14.62 \%$ & 5.5 & 1.06433 & 58.5 & 32.5 \\
\hline 93.2 & 79.4 & 13.8 & $16.14 \%$ & 7.7 & 1.09006 & 59.4 & 33.8 \\
\hline 95.4 & 81.9 & 13.5 & $15.79 \%$ & 9.9 & 1.11579 & 61.9 & 33.5 \\
\hline 87.5 & 79 & 8.5 & $9.94 \%$ & 2 & 1.02339 & 59 & 28.5 \\
\hline 89.3 & 79.3 & 10 & $11.70 \%$ & 3.8 & 1.04444 & 59.3 & 30 \\
\hline 91.5 & 79.5 & 12 & $14.04 \%$ & 6 & 1.07018 & 59.5 & 32 \\
\hline 93.9 & 80.9 & 13 & $15.20 \%$ & 8.4 & 1.09825 & 60.9 & 33 \\
\hline 95.5 & 82.3 & 13.2 & $15.44 \%$ & 10 & 1.11696 & 62.3 & 33.2 \\
\hline
\end{tabular}

\subsection{Effect of Elongation at Heat-setting for Measurement after Dyeing}

According to the Figure 3, shrinkage after dyeing $\left(\mathrm{S}_{\mathrm{AD}}\right)$ increases with the elongation and $\mathrm{d} S_{A D} /$ de reduces with the elongation after heat-setting. It was observed that different elongations at heat- setting resulted in a measurement fluctuation of nearly $5 \mathrm{~cm}$ after dyeing in the same batch. Since dyeing temperature is above the $\mathrm{T}_{\mathrm{g}}$ of polyester, dyeing and tumble-drying processes reduce the hard-yarn resistance due to agitation and permit the spandex recovery forces to further compress the knit loops. This process can result in unacceptable and high levels of shrinkages.

Moreover, after-dye shrinkage $\left(S_{A D}\right)$ is higher than thermal shrinkage $\left(S_{H T}\right)$ due to heat-setting. According to Figure 3 , it is possible to conclude that a higher elongation at heat-setting results in a higher shrinkage after dyeing; consequently, Table 3 depicts that a higher elongation at heat-setting results in a higher measurement after dyeing. These results can be attributed to morphological changes induced on polyester/ spandex yarns after heat-setting and shift in equilibrium states during the subsequent processes of heat-setting and dyeing. The morphological changes are induced due to strain-induced crystallisation of spandex and thermoelastic behaviour of polyester. However, after heat-setting, it was observed a new equilibrium state in dimensions, in which loops are compressed more than that of dry relaxation. The shift in equilibrium states due to heat-setting is attributed to thermal shrinkage of the knitted tubes. Moreover, after dyeing and tumble drying, there is a another shift in equilibrium state, in which loop are further compressed than that of heat-setting. 


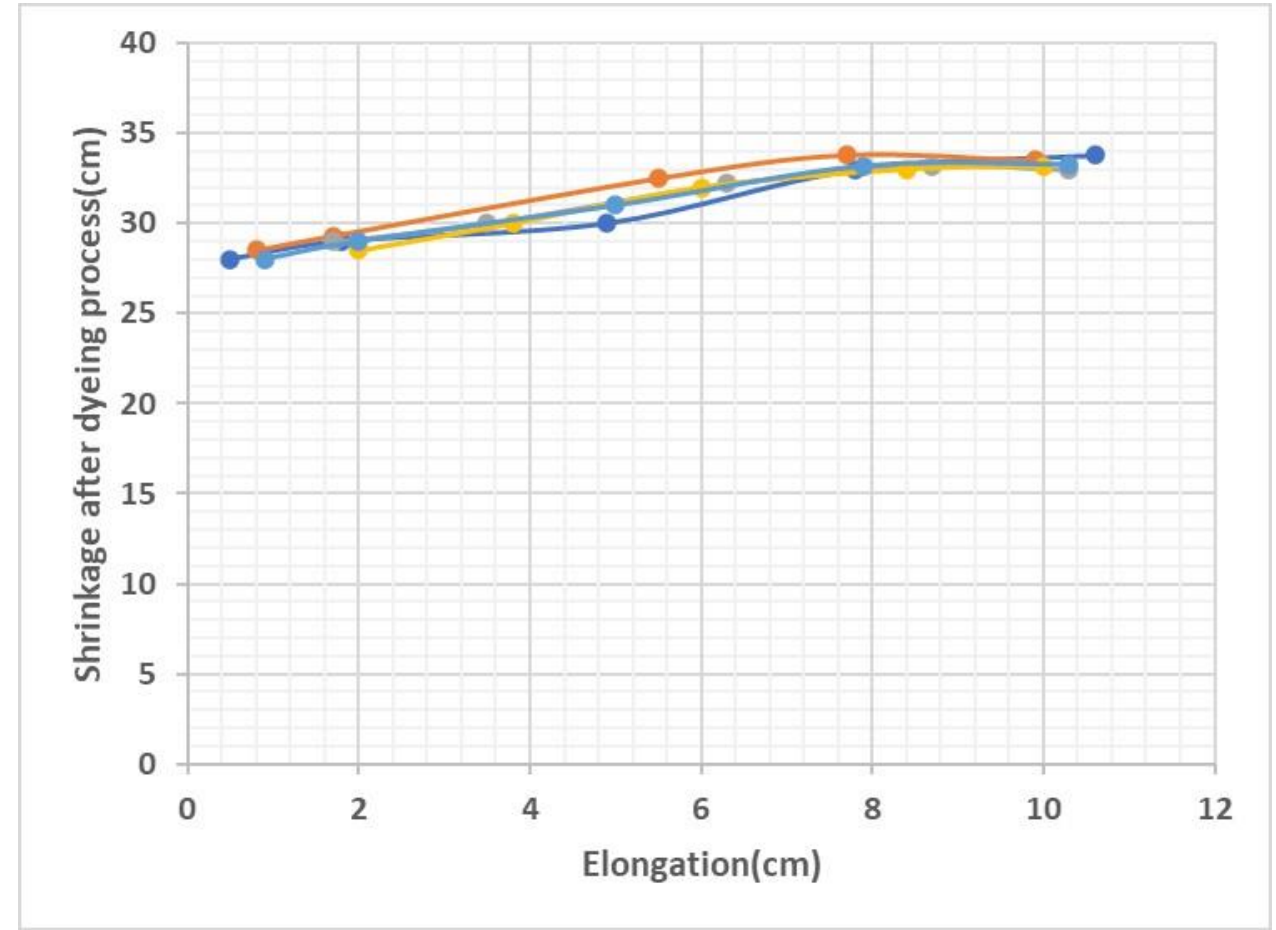

Figure 3: The effect of elongation on shrinkage after dyeing and tumble drying

\subsection{Measurement Adjustment Process from Pilot to Bulk}

The time taken to complete the knitting process of the pilot run is approximately 20 hours. However, it takes nearly 115 hours to complete a batch in the bulk production. The relaxation shrinkage of pilot run is approximately $4 \mathrm{~cm}$ whereas the relaxation shrinkage of bulk is $8 \mathrm{~cm}$, which is almost doubled. As per Figure 4, the relaxed measurement before heat-setting is $90.8 \mathrm{~cm}$ in the pilot which represents to an approximation of $30 \mathrm{~cm}$ shrinkage after dyeing (referring Table 3). During the production, due to the shrinkage variation of $8 \mathrm{~cm}$ within the same batch, the shrinkage of the garments is between $28 \mathrm{~cm}$ and $34 \mathrm{~cm}$ (refer Table 3). This variation of shrinkage represents a variation in measurement in the bulk from $58 \mathrm{~cm}$ to $62.8 \mathrm{~cm}$. Since measurement adjustments are carried out based on the pilot run results, before wash measurement at heat -setting should take into account with respect to an intermediate-state on the relaxation curve. Following this method, it is possible to get the measurements in the batch within the (+) and (-) tolerance level; garments which knit last will fall in the $(+)$ tolerance, and garments which knit first will fall in the (-) tolerance. 


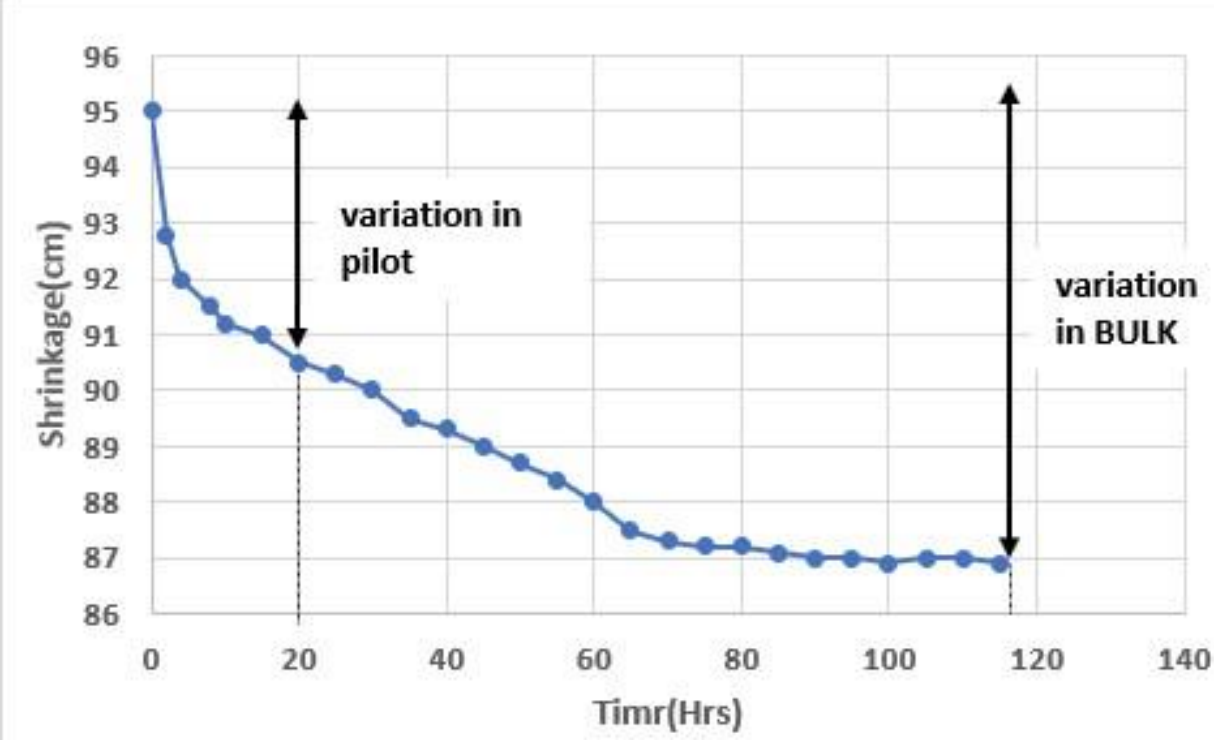

Figure 4: Dry relaxation in pilot run and bulk

\subsection{Accelerate Dry Relaxation by Analysing the Thermoelastic behaviour of Knitted Tubes}

According to Figure 5, $\mathrm{d} L_{l} / \mathrm{dt}$ (gradient of dry relaxation) at $22^{\circ} \mathrm{C}$ is greater than $\mathrm{d} L_{l} / \mathrm{dt}$ at $32^{0} \mathrm{C}$ during the first 10 hours and hence fabric reaches to the equilibrium condition within 40 hours. Moreover, as per Figure 5, specimens reach to $89 \mathrm{~cm}$ within 10 hours at $32^{\circ} \mathrm{C}$. By comparing the results in Table 3, for Tubes which relaxe beyond $89 \mathrm{~cm}$, it is possible to maintain measurement variation within $1.5 \mathrm{~cm}$ difeerence after dyeing. Therefore, the problem with the measurement deviation in the batch after dyeing can be minimised by accelerating the dry relaxation ; in other words, increase in temperature in the batch preparation area will make the bulk reach to an equilibrium state within a short period of time.

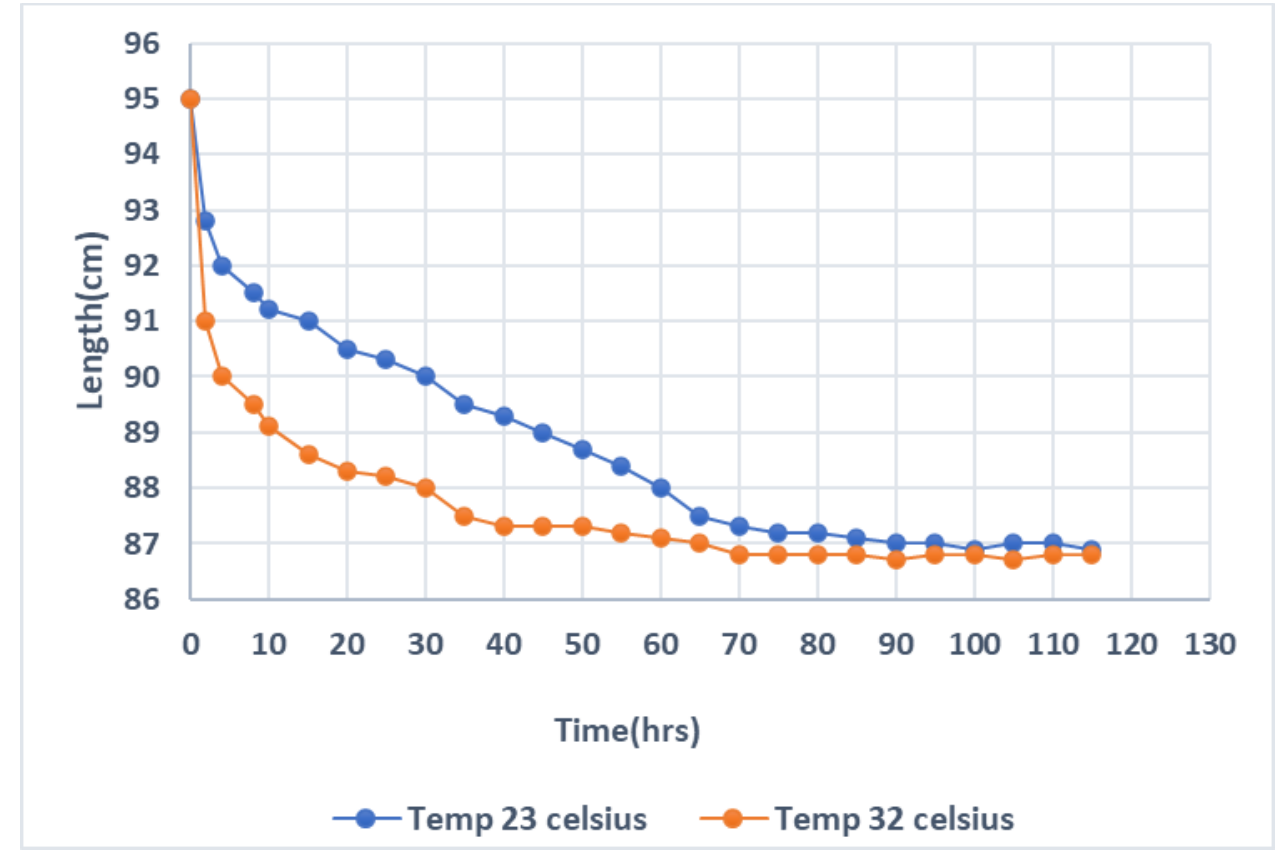

Figure 5: Dry relaxation at $22^{\circ} \mathrm{C}$ and $32^{\circ} \mathrm{C}$

In previous studies, authors have analysed the static thermoelastic behaviour of spandex, the relationship between $\sigma$ (stress) and T(temperature) at a specific elongation and the relationship between $\mathrm{d} \sigma / \mathrm{dT}$ and $\lambda$ (extension ratio). Strain-induced 
crystallisation of spandex has a positive influence on thermal contraction. Marchessault et al. (1985) analysed the dynamic thermoelastic behaviour of thermoplastic elastomers at a temperature profile below $T_{\mathrm{g}}$. Thermoelastic studies performed on spandex films showed an increase in slope of $\mathrm{d} \sigma / \mathrm{dT}$ with $\lambda$ (extension ration) which was attributed to the influence of crystalline orientation due to thermoelastic response (Senthilkumar et al., 2011). The rapid drop in slope of $\mathrm{d} \sigma / \mathrm{dT}$ with $\lambda$ during decreasing elongation was attributed to the reversal processs of strain-induced crystallisation. As per the researcher, the stress induced at a given extension increases with the temperature in the elastic region. Therefore, increase in temperature accelerates the relaxation of spandex by increasing the stress recovery at a given elongation. However, without the assistance of plated hard yarn, fabric cannot shrink at a rapid slope by increasing the temperature. From the experiment results, the rapid slope in $\mathrm{d} L_{l} / \mathrm{dt}$ with temperature at higher elongations reveals that polyester material in the composition has a similar thermoelastic behaviour as spandex. Therefore, the rapid slope of dry relaxation at $32^{\circ} \mathrm{C}$ is attributed to static thermoelastic behaviour of both spandex and polyester.

\section{CONCLUSIONS}

- A higher tensile strain produces a high strain energy and thereby increases the stress relaxation. The rapid slope of relaxation shrinkage is attributed to tensile resilience of polyester and high elastic recovery property of spandex material in the composition. Dry relaxation creates a significant deviation in lengthwise measurement (wale direction) of the bulk where garments are at different elongations prior to heat-setting process.

- The analysis of thermal shrinkage of polyester/spandex plated knit system suggests that thermal shrinkage increases with the increase in elongation, and the gradient of thermal shrinkage reduces with the increase in elongation during the heat-setting process.

- The elongation at heat-setting determines the after-dye measurement. A higher elongation at heat-setting resulted in a higher measurement after dyeing. Moreover, $8 \mathrm{~cm}$ difference in measurement at heat-setting resulted a measurement fluctuation of $4.5 \mathrm{~cm}$ difference after dyeing.

- Increase in temperature from $22^{\circ} \mathrm{C}$ to $32^{\circ} \mathrm{C}$ accelerated the dry relaxation of the knitted tubes, and specimens relaxed beyond $89 \mathrm{~cm}$ within 10 hours. The acceleration of relaxation is attributed to thermoelastic behaviour of spandex and polyester material in the composition. By accelerating the relaxation, the deviation of measurement at heat-setting was reduced by a significant margin. Specimens which relaxed beyond $89 \mathrm{~cm}$ fluctuated within $1.5 \mathrm{~cm}$ after dyeing. Therefore, this research suggests that increase in temperature brings a higher portion of tubes within a small fluctuation during the dry relaxation whih will reduce the measurement deviation at heat-setting and hence after dyeing.

- Pilot run is suggested to conduct by analysing the dry relaxation curve of the knitted tubes. The heat-setting process of the pilot run should be performed based on the measurement of an intermediate state on the dry relaxation curve This process will bring knitted tubes which knit first and last inside the tolerance level after dyeing.

\section{ACKNOWLEDGEMENT}

I would like to express my gratitude towards the management of Linea Intimo (pvt) Ltd. for providing their services to execute this research successfully. 


\section{REFERENCES}

1. Bandari, S. S. M., Asayesh, A., \& Latifi, M. (2020). The Effect of Fabric Structure and Strain Percentage on the Tensile Stress Relaxation of Rib Weft Knitted Fabrics. Fibers and Polymers, 21, 921-929. doi:10.1007/s12221-020-9450-6

2. Eltahan, E. A. E., Sultan, M., \& Mito, A. B. (2016). Determination of loop length, tightness factor and porosity of single jersey knitted fabric. Alexandria Engineering Journal, 55(2), 851-856. doi:10.1016/j.aej.2016.02.006

3. V.Bhasker, S.Priyanka, S.S.Sukeerthi, V.Satya Radhika, G.Sindhura Reddy \& Suman Mokenapalli, "Formulation and Evaluation of Finger Millet Based Extruded Product (Finger Millet Shells) «, IMPACT: International Journal of Research in Engineering \& Technology (IMPACT: IJRET), Vol. 5, Issue 10, pp. 9-20

4. Ertekin, G., \& Marmarali, A. (2016). The effect of heat-setting conditions on the performance characteristics of warp knitted spacer fabrics. Journal of Engineered Fibers and Fabrics, 11(3), 64-71. doi:10.1177/155892501601100309

5. Fatkić, E., Geršak, J., \& Ujević, D. (2011). Influence of knitting parameters on the mechanical properties of plain jersey weft knitted fabrics. Fibres and Textiles in Eastern Europe, 19(5), 87-91.

6. Renuka Chauhan \& R. C. Srivastava, " Dielectric Study of Bazr0.5Ti0.5O3 Ferroelectric Relaxor Ceramic », International Journal of Electronics and Communication Engineering (IJECE), Vol. 4, Issue 5, pp. 1-8

7. Gacén, J., Cayuela, D., Maulo, J., \& Gacén, I. (2002). Physico-chemical analytical techniques for evaluation of polyester heatsetting. Journal of the Textile Institute, 93(1), 29-42. doi:10.1080/00405000208630550

8. Gowers, C. N., \& Hurt, F. N. (1978). 14-The wet-relaxed dimensions of plain-knitted fabrics. Journal of the Textile Institute, 69(4), 108-115. doi:10.1080/00405007808631428

9. G. A Isola \& D. A Ajadi, «Estimation of Natural Radioactivity Levels in Beach Sands from Lagos, South-Western Nigeria », BEST: International Journal of Humanities, Arts, Medicine and Sciences (BEST: IJHAMS), Vol. 3, Issue 7, pp.45-50

10. Gupta, V. B. (2001). Heat setting. Journal of Applied Polymer Science. 83, 586-609, https://doi.org/10.1002/app.2260

11. Hurd, J. C. H., \& Doyle, P. J. (1953). Fundamental aspects of the design of knitted fabrics, 44(8), P561-P578. Journal of the Textile Institute Proceedings. doi:10.1080/19447015308687861

12. Jayawardana, T. S. S., Fernando, E. A. S. K., Wijesena, G. H. D., \& Kuruppu, R. (2016). Development an Effective Quick Fabric Relaxation Machine. International Journal of Engineering Trends and Technology, 42(6), 310-318. doi:10.14445/22315381/ijett-v42p256

13. Lau, K. L., \& Dias, T. (1994). Knittability of High-modulus Yarns. The Journal of The Textile Institute, 85(2), 173-190. doi: $10.1080 / 00405009408659018$

14. Marchessault, R. H., Bluhm, T. L., Gharapetian, H. M., \& Sundararajan, P. R. (1985). Influence of molecular structure on the dynamic thermoelasticity of polyphasic polymer systems. Canadian Journal of Chemistry, 63(1), 189-195. doi:10.1139/v85-030

15. Mody, R., Lofgren, E. A., \& Jabarin, S. A. (2001). Shrinkage behaviour of oriented poly(ethylene terephthalate). Journal of Plastic Film and Sheeting, 17(2), 152-163. doi:10.1106/YOFP-L790-OKEE-YV6J

16. Munden, D. L. (1959). 26-The geometry and dimensional properties of plain-knit fabrics. Journal of the Textile Institute Transactions, 50(7), T448-T471. doi:10.1080/19447025908659923

17. Pavko-Cuden, A., Hladnik, A., \& Sluga, F. (2013). Loop length of plain single weft knitted structure with elastane. Journal of Engineered Fibers and Fabrics, 8(2), 110-120. doi:10.1177/155892501300800214 
18. Perera, H. A. A. E., \& Lanarolle, W. D. G. (2020). Comparative study on the thermal shrinkage behaviour of polyester yarn and its plain knitted fabrics. The Journal of The Textile Institute. doi:10.1080/00405000.2020.1729650

19. Prakash, C., \& Thangamani, K. (2010). Establishing the effect of loop length on dimensional stability of single jersey knitted fabric made from cotton/lycra core spun yarn. Indian Journal of Science and Technology, 3(3), 287-289. doi:10.17485/ijst/2010/v3i3/29698

20. Sanjari, H. R., Semnani, D., \& Sheikhzadeh, M. (2011). Investigating the performance of various relaxation processes on the surface regularity and dimensional properties of plain knitted fabrics using the image processing technique. Fibres and Textiles in Eastern Europe, 36-42.

21. Senthilkumar, M., Anbumani, N., \& De Araujo, M. (2011). Elastic properties of spandex plated cotton knitted fabric. Journal of the Institution of Engineers (India), Part TX: Textile Engineering Division, 92, 1-5.

22. Manal A. Seif \& Shaimaa M. Ahmed, "Impactof Printing Techniquesonthe Properties Behaviourof Seamed Textile Fabrics », International Journal of GeneralEngineering and Technology (IJGET), Vol. 7, Issue2,pp. 1-16

23. Senthilkumar, M., Sounderraj, S., \& Anbumani, N. (2012). Effect of spandex input tension, spandex linear density and cotton yarn loop length on dynamic elastic behaviour of cotton/spandex knitted fabrics. Journal of Textile and Apparel, Technology and Management, 7(4), 1-16.

24. O'Dottaviano, F., Cambrils, T., \& Lamia L A. Stretch fabrics with improved heat-setting properties. US 7, 943, 536, B2, filed August 12, 2005, and issued May 17, 2011.

\section{AUTHOR PROFILE}



I am a Textile and Process Engineer who was graduated from University of Moratuwa. My knowledge spans in all areas of the textile industry such as garment manufacturing, textile colouration, fabric formation (weaving and knitting), yarn formation (wet and dry spinning), fiber science, production planning and controlling, operational research, apparel marketing and merchandising, textile pretreatment processes, wet and dry processing of garments and fabric/garment testing. 
